\title{
3 Research Square

\section{The Effect of Preemptive Local Anesthesia on Postoperative Pain Following Vaginal Hysterectomy: A Randomized Controlled Trial}

Ohad Feldstein ( $\square$ ohad60@gmail.com )

Wolfson Medical Center

Elad Barber

Wolfson Medical Center

Liliya Tamayev

Wolfson Medical Center

Alexander Condrea

Wolfson Medical Center

Ehud Grinstein

Wolfson Medical Center

Ron Sagiv

Wolfson Medical Center

Inna Wolfson

Wolfson Medical Center

Jacob Bar

Wolfson Medical Center

Shimon Ginath

Wolfson Medical Center

Ohad Gluck

Wolfson Medical Center

\section{Research Article}

Keywords: anesthesia, hysterectomy, PREEMPTIVE

Posted Date: February 5th, 2021

DOI: https://doi.org/10.21203/rs.3.rs-153185/v1

License: (c) (1) This work is licensed under a Creative Commons Attribution 4.0 International License.

Read Full License 


\section{Abstract}

Introduction: The effect of local anesthesia on postoperative pain, in operations performed vaginally has been scarcely studied. Although the results were encouraging, the studies populations were relatively small, and the indications for hysterectomy were not homogenous.

We aimed to study the effect of preemptive local anesthetic on postoperative pain following vaginal hysterectomy.

Methods: This was a double-blinded, randomized, controlled trial. Women who undergone elective vaginal hysterectomy were included. Solutions of either Bupivacaine-Hydrochloride $0.5 \%$, or Sodium-Chloride $0.9 \%$ as a placebo, were prepared prior to surgery, according to randomization. The chosen solution was injected before incision, in a circumferential manner, to the cervix. The amount of fluid administered was $10 \mathrm{ml}$. When colporrhaphy was also performed, an additional $5 \mathrm{ml}$ of solution were injected in the midline of the vaginal wall prior to each incision line. We conformed to the CONSORT recommendations.

By utilizing the $10 \mathrm{~cm}$ Visual-analogue-scale (VAS) we assessed post-operative pain at rest at 3, 8, and 24 hours, and during ambulation at 8 and 24 hours.

Results: A total of 30 women were included in each group. The level of postoperative pain, as assessed by VAS, was not significantly different between the groups, in all points of time. In addition, there was no difference between the groups in opioid based analgesics during recovery, nor in postoperative analgesic use.

Conclusion: Preemptive local anesthesia was not shown to be efficient in reducing postoperative pain after vaginal hysterectomy.

\section{Introduction}

The concept of fast-track surgery has been evolved and can be attributed to decreasing postoperative hospitalization and morbidity [1, 2]. It has already been demonstrated that preoperative local anesthesia, into incision sites, reduces both subjective post-operative pain, and objectively, the use of opioid-based analgesia after gynecological laparoscopies [1, 2].

It has been shown that nociceptive stimuli can alter the electrophysiological processes in the neurons [3]. This alteration results in a lower pain threshold and an increased response to pain stimuli. By infiltrating a local anesthetic before the incision is made, these effects are avoided [4].

This issue is of a great significance in perioperative management of older patients, as it has been reported that aging reduces pain sensitivity for lower pain intensities [5].

Hysterectomy is the second most common surgical procedure among women in the United States [6, 7]. A recent Cochrane review [7] demonstrated that vaginal hysterectomy proved to be the superior procedure 
since it was associated with the quickest return to normal activities, earliest discharge from hospital, and had the shortest operation time compared to both laparoscopic and abdominal hysterectomy.

Only few studies have investigated the effect of local anesthesia on postoperative pain, in operations performed vaginally [8-13]. Of them, only one study was conducted among patients who had undergone vaginal hysterectomy that included patients with pelvic organ prolapse (POP) [12]. Although the results were encouraging, the sample size was relatively small, and the indications for hysterectomy were not homogenous.

Therefore, our aim was to study whether preemptive local anesthetic reduces postoperative pain or use of analgesics following POP-indicated vaginal hysterectomy.

\section{Results}

During the study period, 75 women were eligible for participation, of them, five were not meeting the inclusion criteria, and four women refused to participate. After enrollment, six women did not receive the injection due to technical reasons. Ultimately, a total of 60 women completed full pain assessment (Fig. 1). All women returned for a follow up visit. No malignancies were detected on final pathological examination. In addition, no late complication were noticed.

All the patients undergone vaginal hysterectomy indicated by uterine prolapse stage $2-4$. The mean age at the medication and placebo groups was $67.3 \pm 8.2$ and $66.7 \pm 8.4$ years old, respectively $(p=0.9)$. Background characteristics were similar between the groups (Table 1). There were no significant differences in surgical characteristics between the groups (Table 2). One major complication occurred in the medication group, a large pelvic hematoma which was detected by computed tomography scan, due to complaints of pelvic pain, and was managed conservatively. 
Table 1

Background characteristics of the study groups

\begin{tabular}{|llll|}
\hline & $\begin{array}{l}\text { Medication group } \\
(\mathbf{n}=\mathbf{3 0})\end{array}$ & $\begin{array}{l}\text { Placebo group } \\
(\mathbf{n}=\mathbf{3 0})\end{array}$ & p-value \\
\hline Age (years) & $67.3 \pm 8.2$ & $66.7 \pm 8.4$ & 0.9 \\
\hline Body mass index $\left(\mathrm{kg} / \mathrm{m}^{2}\right)$ & $23.6 \pm 10.2$ & $27.3 \pm 3.6$ & 0.3 \\
\hline Gravity & $4.0 \pm 1.7$ & $3.9 \pm 1.6$ & 0.7 \\
\hline Parity & $2.8 \pm 1.0$ & $2.7 \pm 1.0$ & 0.8 \\
\hline Smoking & $2(7.1)$ & $5(17.9)$ & 0.4 \\
\hline Previous abdominal surgeries & $11(36.7)$ & $11(36.7)$ & 1.0 \\
\hline Apical prolapse stage 3-4 & $25(83.3)$ & $26(86.7)$ & 0.9 \\
\hline Uterine size $\left(\mathrm{cm}^{3}\right)$ & $7.8 \pm 5.0$ & $10.6 \pm 7.6$ & 0.1 \\
\hline Data are presented as mean \pm standard deviation, or $\mathrm{n}(\%)$. & \\
\hline
\end{tabular}

Table 2

Surgical characteristics according to study groups

\begin{tabular}{|llll|}
\hline & $\begin{array}{l}\text { Medication group } \\
(\mathbf{n}=\mathbf{3 0})\end{array}$ & $\begin{array}{l}\text { Placebo group } \\
(\mathbf{n}=\mathbf{3 0})\end{array}$ & p-value \\
\hline Surgery length (minutes) & $90 \pm 3030$ & $81 \pm 29$ & 0.9 \\
\hline Major complications & $0(0)$ & $1(3.6)$ & 1.0 \\
\hline Posterior colporrhaphy & $22(73.3)$ & $23(76.7)$ & 0.2 \\
\hline Anterior colporrhaphy & $27(90.0)$ & $27(90.0)$ & 1.0 \\
\hline Anterior and posterior colporrhaphy & $21(70.0)$ & $22(73.3)$ & 0.4 \\
\hline Anti-incontinence procedure* & $25(83.3)$ & $27(90.0)$ & 0.7 \\
\hline Data are presented as mean \pm standard deviation, or $\mathrm{n}(\%)$. & \\
\hline *Trans obturator tape & & \\
\hline
\end{tabular}

Table 3 depicts the postoperative pain levels in the study groups. During the first 24 hours after surgery the mean levels of pain were low to moderate (up to 5.1), at all points of measurement. Although a slight trend was observed between the groups regarding the primary outcome (abdominal pain during ambulation at 8 hours postoperatively), it was not statistically significant (medication group $4.1 \pm 3.0$, placebo group $4.8 \pm 3.5, p=0.45$ ). Moreover, no significant differences were observed between the groups 
at other points of time, regardless of pain location (abdomen or vagina) or type (at rest or during ambulation).

Table 3

Postoperative pain levels according to study groups (Assessed by VAS score)

\begin{tabular}{|llll|}
\hline & \multicolumn{1}{|c|}{$\begin{array}{l}\text { Medication group } \\
(\mathbf{n}=\mathbf{3 0})\end{array}$} & $\begin{array}{l}\text { Placebo group } \\
(\mathbf{n}=\mathbf{3 0})\end{array}$ & p-value \\
\hline Pain level at 3 hours after surgery & & \\
\hline Abdomen - rest & $4.9 \pm 3.0$ & $5.1 \pm 3.3$ & 0.8 \\
\hline Vagina- rest & $4.4 \pm 3.9$ & $3.9 \pm 3.9$ & 0.6 \\
\hline Pain level at 8 hours after surgery & & \\
\hline Abdomen - rest & $3.5 \pm 2.9$ & $3.7 \pm 3.0$ & 0.7 \\
\hline Vagina- rest & $3.2 \pm 3.2$ & $4.2 \pm 3.2$ & 0.3 \\
\hline Abdomen - ambulation & $4.1 \pm 3.0$ & $4.8 \pm 3.5$ & 0.4 \\
\hline Vagina- ambulation & $3.5 \pm 3.4$ & $5.0 \pm 3.8$ & 0.1 \\
\hline Pain level at 24 hours after surgery & & \\
\hline Abdomen - rest & $4.0 \pm 2.8$ & $4.6 \pm 2.8$ & 0.4 \\
\hline Vagina- rest & $3.0 \pm 2.9$ & $4.2 \pm 3.3$ & 0.2 \\
\hline Abdomen - ambulation & $3.8 \pm 3.4$ & $4.9 \pm 3.2$ & 0.3 \\
\hline Vagina- ambulation & $3.5 \pm 3.4$ & $5.0 \pm 3.4$ & 0.1 \\
\hline Data are presented as mean \pm standard deviation. & \\
\hline
\end{tabular}

After performing analysis of postoperative analgesics use, we did not observe any differences between the groups, neither for analgesic use in general nor for opioid-base analgesic use specifically (Table 4). There was also no between groups difference in rate of nausea or vomiting. 
Table 4

Postoperative assessment and analgesics requirements according to study groups

\begin{tabular}{|c|c|c|c|}
\hline & $\begin{array}{l}\text { Medication group } \\
(n=30)\end{array}$ & $\begin{array}{l}\text { Placebo group } \\
(n=30)\end{array}$ & p-value \\
\hline \multicolumn{4}{|c|}{ Assessment and analgesics requirements at 3 hours after surgery } \\
\hline Nausea/ vomiting & $6(20.0)$ & $6(20.0)$ & 1.0 \\
\hline Analgesia- all types & $10(33.3)$ & $12(40.0)$ & 0.4 \\
\hline Analgesia- opioids & $0(0)$ & $4(13.3)$ & 0.1 \\
\hline \multicolumn{4}{|c|}{ Assessment and analgesics requirements at $\mathbf{8}$ hours after surgery } \\
\hline Nausea/ vomiting & $2(6.7)$ & $5(16.7)$ & 0.4 \\
\hline Analgesia- all types & $19(63.3)$ & $12(40.0)$ & 0.5 \\
\hline Analgesia- opioids & $0(0)$ & $0(0)$ & 1.0 \\
\hline \multicolumn{4}{|c|}{ Assessment and analgesics requirements at 24 hours after surgery } \\
\hline Nausea/ vomiting & $1(3.3)$ & $3(10.0)$ & 0.3 \\
\hline Analgesia- all types & $14(46.7)$ & $9(30.0)$ & 0.4 \\
\hline Analgesia- opioids & $1(3.3)$ & $1(3.3)$ & 1.0 \\
\hline
\end{tabular}

\section{Discussion}

In this study, we found that both medication and placebo groups had similar levels of postoperative pain and analgesic requirements, at all points of time, during the first 24 hours following vaginal hysterectomy. However, we did find that postoperative levels of pain, during the first 24 hours after vaginal hysterectomy, are low to moderate.

In contrary to our results, other studies demonstrated that paracervical block with local anesthesia was effective in reducing pain scores for the first several hours and opioid use during the first 24 hours postoperatively: O'Neal et al. [13] compared preemptive local anesthetics using $20 \mathrm{ml} 0.5 \%$ Bupivacaine with Epinephrine to $\mathrm{NaCl}$ in 20 women undergoing vaginal hysterectomy. Total Morphine use and pain scores were significantly less in the Bupivacaine group.

Long et al. [12] compared preemptive local analgesia in 90 women undergoing vaginal hysterectomy. Local analgesia consisted of $20 \mathrm{ml}$ of 0.5\% Bupivacaine with 1:200,000 Epinephrine injected in a paracervical-type fashion. Total narcotic use and pain scores were significantly less in the Bupivacaine group versus the placebo group. 
Kristensen et al. [8] compared the effect of local infiltration of Ropivacaine $\mathrm{Vs}$. $\mathrm{NaCl}$, in women undergoing posterior wall repair and perineorrhaphy. Postoperative pain at rest, during coughing and movement was significantly reduced in the Ropivacaine group.

Hristovska et al. [10] found that postoperative pain after vaginal hysterectomy at rest and during movements was significantly reduced, using Ropivacaine.

There are several possible explanations for the conflicting results: First, the drug dose we used $(10 \mathrm{ml}$ of Bupivacaine hydrochloride $0.5 \%$ for paracervical block \pm up to $10 \mathrm{ml}$ of Bupivacaine hydrochloride $0.5 \%$ in cases of additional colporrhaphy) was lower than that used in the trials that demonstrated differences in pain and opioid use. It is also important to mention that Bupivacaine carries the highest risk of cardiovascular toxicity among the various local anesthetics available [14], and it is therefore possible that the threshold for adequate postoperative pain relief could not be reached due to maximal-dose limitation. However, most of the women in the medication group received a total of $20 \mathrm{ml}$ solution, an amount which have been reported as sufficient, in previous studies $[12,13]$.

Second, the current study population differ from previous studies by the homogeneity of surgery indication and technique. It is possible that uterine prolapse indicated vaginal hysterectomy for women with normal-sized uterus, is not painful enough to reach statistical differences between groups with regards to postoperative pain. Lastly, the elimination half-life $(T 1 / 2 \beta)$ of Bupivacaine is about 210 minutes. It is possible that the effect of preemptive local anesthetics does not last long enough to reduce pain in the first 24 hours after surgery [15]. Nevertheless, it is important to emphasize, that the preemptive local anesthesia effect aims to prevent the neural sequel, caused by the pain stimulation during the surgery, which lasts much less than 3 hours.

This study has several strengths. First, to the best of our knowledge, this is the first study to focus in elderly women. In addition, all the patients had the same indication for surgery- uterine prolapse, a fact that adds to homogeneity. It seems that the patient's age, as well as the indication for hysterectomy plays a crucial role in pain aggravation and management; Therefore, the homogeneity of the study group is valuable. Second, the randomized, double- blinded, design minimizes the risk for potential bias. Lastly, we investigated the effect of preemptive analgesia on postoperative level of pain, as well as medications use, including the demand for opioid-based analgesics.

Our study has few limitations. First, the effect of the preemptive local anesthesia on the time elapsed from surgery to full mobility was not evaluated. However, as thromboembolic events are a major concern in non-mobile patients, none has occurred among the study population.

Second, only short-term outcomes were assessed in the current study, while full recovery period which last until the patient returns to full activity, was not assessed. However, since no difference was detected in the first 24 hours, it is safe to assume that no differences would have been found later on. 
In conclusion, it appears that preemptive administration of local analgesia does not affect the level of postoperative pain in POP indicated vaginal hysterectomy or the use of postoperative analgesics. Further studies are needed to investigate the use of other medications, doses, and routes of administration in reducing postoperative pain.

\section{Methods}

Participants were recruited from a single-center gynecologic department at the Edith Wolfson Medical center, Holon, Israel, from September 1st, 2017 until November 30th, 2019. Inclusion criteria included women in general good health (defined as American Society of Anesthesiologists grade 1-2) who underwent elective vaginal hysterectomy indicated by pelvic organ prolapse (POP). Exclusion criteria included chronic pain requiring use of analgesics, allergy to Bupivacaine hydrochloride, additional concurrent abdominal/ laparoscopic procedures, active pelvic infection, or malignancy. Post-assignment exclusion was executed in cases of conversion to laparotomy/ laparoscopy.

This study was approved by the Wolfson medical center review board (approval number 0156-17-WOMC, dated 17/08/2017) and written informed consent was obtained from all subjects participating in the trial. The trial was registered prior to patient enrollment at clinicaltrials.gov (NCT03268525, Principal investigator: Ohad Gluck, Date of registration: 31/08/2017). This manuscript adheres to the applicable CONSORT guidelines [16].

This is a double-blinded study, with parallel assignments, to one of two groups:

The medication group- Bupivacaine hydrochloride $0.5 \%$, and the placebo group-Sodium Chloride ( $\mathrm{NaCl})$ $0.9 \%$.

At the day of surgery, women were randomly assigned to one of the study groups. In order to ensure allocation concealment the random allocation sequence was kept with the lead author (OG), which was not a part of the recruiting, operating, or pain assessment team.

An assigned nurse (IW) prepared syringes prior to each surgery, outside of the operation room, according to the randomization list, and unlabeled syringes were provided to the surgeons upon commencing surgery. The content of each syringe was written in the concealed list only. Women, surgeons, anesthesiologists, and pain assessment researchers were all blinded to participants' allocations.

The syringes contained $10 \mathrm{ml}$ of Bupivacaine hydrochloride $0.5 \%$ or $\mathrm{NaCl} 0.9 \%$ as placebo. A total of $8 \mathrm{ml}$ of solution was injected before incision, to the sub-mucosa layer of the exo-cervix, $2 \mathrm{~cm}$ above the external OS, in a circumferential manner. In addition, $1 \mathrm{ml}$ of the solution was injected in each resection line (sacro-uterine and cardinal ligaments), adding up to a total of $10 \mathrm{ml}$. In case of performing additional anterior/ posterior colporrhaphy, an extra $5 \mathrm{ml}$ of solution were injected to the cutting line at the anterior/ posterior vaginal wall, respectively, prior to incision (additional $5 \mathrm{ml}$ for anterior or posterior repair and 10 $\mathrm{ml}$ if both were performed). Based on previous studies [12,13], a total amount of $10-20 \mathrm{ml}$ of 
Bupivacaine hydrochloride $0.5 \%$ or $\mathrm{NaCl} 0.9 \%$, was injected prior to surgery. No vasoconstrictors were injected. After injection, a 5 minutes pause was conducted, prior to cutting, for reaching maximal effect of anesthesia.

All surgeries were performed under general anesthesia. All patients received standardized anesthetic induction and maintenance. Patients were premedicated with $2 \mathrm{mg}$ intravenous (IV) midazolam. General anesthesia was induced with $2 \mathrm{mg} / \mathrm{kg}$ IV Propofol, $2 \mathrm{mg} / \mathrm{kg}$ IV Fentanyl citrate, and $0.6 \mathrm{mg} / \mathrm{kg} \mathrm{IV}$ Rocuronium Bromide. General anesthesia was maintained with an infusion of $50-150 \mathrm{mg} / \mathrm{kg} / \mathrm{minute}$ IV Propofol, combined with inhaled Sevoflurane and oxygen. Under general anesthesia with endotracheal intubation, the patient was positioned properly and draped. Prior to surgery, second generation Cephalosporins was routinely administered, as part of our departmental protocols, and Clexane (Enoxaparin) was administered when indicated.

All procedures were performed by one of two senior surgeons (authors A.C and S.G). Vaginal hysterectomy was conducted with concomitant McCall Culdoplasty, for addressing apical support, in order to prevent recurrence of POP [17]. Additional procedures (colporrhaphy and/or mid-urethral sling), if conducted, were performed after the hysterectomy was completed.

Pain medication in the recovery room, was provided by the attending nurses upon demand, according to the following regime:

a. First-line: $1 \mathrm{~g}$ IV Paracetamol, up to 4 times per day upon demand

b. Second-line: $1 \mathrm{~g}$ oral Dipyrone (not available in the USA), up to 4 times per day upon demand

c. Third-line: Opioid- based medication, either 2-3 mg IV Morphine (As Kalceks, Riga, Latvia) or 2030mg IV Meperidine.

Upon transfer from the recovery room to our department, a uniform, standardized, postoperative painrelief regime was applied for all patients (upon demand), consisting of:

a. First-line: IV Paracetamol 1g, up to 4 times per day

b. Second-line: Oral Dipyrone $1 \mathrm{~g}$, up to 4 times per day

c. Third-line: Intramuscular Diclofenac Sodium $75 \mathrm{mg}$, up to 3 times per day

d. Fourth-line: $100 \mathrm{mg}$ IV Tramadol Hydrochloride (Dexcel, Or-Yehuda, Israel), up to 3 times per day upon demand, usually along with 10mg IV Metoclopramide (Rafa, Jerusalem, Israel).

A urine catheter and vaginal dressing were left at the end of the surgery, and were removed at postoperative day 1 . Patients were discharged 2-3 days postoperatively, and returned for a routine follow-up visit 2-3 weeks after surgery.

Postoperative pain level at rest was evaluated at 3, 8, and 24 hours after surgery, and postoperative pain level during ambulation was evaluated at 8 and 24 hours after surgery. A member of the surgical team who was not involved in the index surgical case administered the pain level assessment. 
Since the visual analog scale (VAS) has been used in prior studies for evaluating similar outcomes [10], a 100-mm VAS was used to grade the level of pain. For assessing pain at rest, patients were asked to grade their level of pain from 0 (no pain) to 10 (worst pain ever experienced) at the abdomen and at the vagina while lying in bed. For assessing pain during ambulation, patients were asked to stand up, walk a few steps, and sit in a chair. Then, they were asked to grade their pain, using the same scale, during ambulation. In addition, patients were also asked whether they have nausea or vomiting, and were evaluated for analgesics requirements.

\section{Primary outcomes}

The primary outcome was the level of abdominal pain during ambulation (according to VAS score) 8 hours after surgery. Based on previous studies, it was estimated that the intervention would cause a $25 \%$ reduction in the primary outcome, as compared to placebo $[10,13]$. In order to attain a power of $80 \%$ and a significance level of 0.05 , the required total sample size was calculated to be 27 women for each group. We included 30 women in each group, in order to make up for any potential loss of follow up. The randomization was performed in blocks of 30 , using an on-line randomization program (http://www.randomization.com).

\section{Secondary outcomes}

Secondary outcomes were levels of pain (abdomen and/or vagina) at other points in time, at rest and/or during ambulation, as well as the demand for postoperative analgesics.

\section{Statistical analyses}

All statistical analyses were conducted using the R Statistical Software, version 3.5.2 (Foundation for Statistical Computing, Vienna, Austria).

We used Analysis of Variance (ANOVA) for continuous variables and the Chi-square or Fisher exact tests for categorical variables.

\section{Declarations}

\section{Author contribution:}

O. G.: This author helped in conceptualization and writing.

O. F.: This author helped in investigation, methodology, and project administration.

E. B.: This author helped in investigation, data curation, review \& editing.

L. T.: This author helped in investigation, data curation, and project administration.

A. C.: This author helped in investigation, data curation, and validation. 
E. G.: This author helped in writing, formal analysis, and review.

I. W.: This author helped in investigation, methodology, and validation.

R. S.: This author helped in investigation and formal analysis.

J. B.: This author helped in supervision, methodology, and validation.

S. G.: This author helped in conceptualization, investigation, and writing.

Additional information:

Financial Disclosures: None.

Conflicts of Interest: None.

Clinical trial identification number: NCT03268525

Date of trial registration $31 / 08 / 2017$

URL of the registration site: https://clinicaltrials.gov

Statistical analyses: R Statistical Software, version 3.5.2, URL https://www.R-project.org.

\section{References}

1. Ghezzi, F. et al. Preemptive port site local anesthesia in gynecologic laparoscopy: A randomized, controlled trial. J Minim Invasive Gynecol. 12, 210-215 https://doi.org/10.1016/j.jmig.2005.03.007 (2005).

2. Ravndal, C. \& Vandrevala, T. Preemptive Local Anesthetic in Gynecologic Laparoscopy and Postoperative Movement-Evoked Pain: A Randomized Trial. J Minim Invasive Gynecol. 23, 775-780 https://doi.org/10.1016/j.jmig.2016.03.009 (2016).

3. Wilder-Smith, O. H. G. Pre-emptive analgesia and surgical pain. Prog Brain Res. 129, 505-524 https://doi.org/10.1016/S0079-6123(00)29037-7 (2000).

4. Joshi, G. P. \& Machi, A. Surgical site infiltration: A neuroanatomical approach. Best Pract Res Clin Anaesthesiol. 33, 317-324 https://doi.org/10.1016/j.bpa.2019.07.017 (2019).

5. Lautenbacher, S., Peters, J. H., Heesen, M., Scheel, J. \& Kunz, M. Age changes in pain perception: A systematic-review and meta-analysis of age effects on pain and tolerance thresholds. Neurosci Biobehav Rev. 75, 104-113 https://doi.org/10.1016/j.neubiorev.2017.01.039 (2017).

6. Moore, B. J., Steiner, C. A., Davis, P. H., Stocks, C. \& Barrett, M. L. Trends in Hysterectomies and Oophorectomies in Hospital Inpatient and Ambulatory Settings, 2005-2013: Statistical Brief \#214. 2006. 
7. Nieboer, T. E. et al. Surgical approach to hysterectomy for benign gynaecologycal disease. Cochrane Database Syst Rev. 3, https://doi.org/10.1002/14651858.CD003677.pub5 (2009). .www.cochranelibrary.com

8. Kristensen, B. B. et al. Local infiltration analgesia in urogenital prolapse surgery: A prospective randomized, double-blind, placebo-controlled study. Acta Obstet Gynecol Scand. 90, 1121-1125 https://doi.org/10.1111/j.1600-0412.2011.01234.x (2011).

9. Fulcher, P. H. et al. Intraoperative utilization of dexamethasone/bupivacaine/gentamicin solution in laparoscopic assisted vaginal hysterectomy and pain management.W V Med J n.d.;110:10-5.

10. Hristovska, A. M. et al. Effect of systematic local infiltration analgesia on postoperative pain in vaginal hysterectomy: A randomized, placebo-controlled trial. Acta Obstet Gynecol Scand. 93, 233238 https://doi.org/10.1111/aogs.12319 (2014).

11. Kuhn, A., Gelman, W., O'Sullivan, S. \& Monga, A. The feasibility, efficacy and functional outcome of local anaesthetic repair of anterior and posterior vaginal wall prolapse. Eur J Obstet Gynecol Reprod Biol. 124, 88-92 https://doi.org/10.1016/j.ejogrb.2005.06.009 (2006).

12. Long, J. B. et al. Randomized trial of preemptive local analgesia in vaginal surgery. Int Urogynecol J. 20, 5-10 https://doi.org/10.1007/s00192-008-0716-6 (2009).

13. O'Neal, M. G., Beste, T. \& Shackelford, D. P. Utility of preemptive local analgesia in vaginal hysterectomy 2003:30-2. doi:10.1016/j.ajog.2003.10.691.

14. Lin, Y. \& Liu, S. S.. Local anesthetics. In: Barash PG, Cullen BF, Stoelting RK et al. Clinical Anesthesia. 8th ed. Philadelphia:Lippincott Wil- liams \& Wilkins; 2017.

15. Becker, D. E. \& Reed, K. L. Local anesthetics: review of pharmacological considerations. Anesth Prog. 59, 90 https://doi.org/10.2344/0003-3006-59.2.90 (2012).

16. Schulz, K. F., Altman, D. G. \& Moher, D. CONSORT 2010 statement: Updated guidelines for reporting parallel group randomized trials. Ann Intern Med. 152, 726-732 https://doi.org/10.7326/0003-4819152-11-201006010-00232 (2010).

17. Alas, A., Chandrasekaran, N. \& Devakumar, H. Advanced uterovaginal prolapse: is vaginal hysterectomy with McCall culdoplasty as effective as in lesser degrees of prolapse ? 2017. doi:10.1007/s00192-017-3436-y.

\section{Figures}




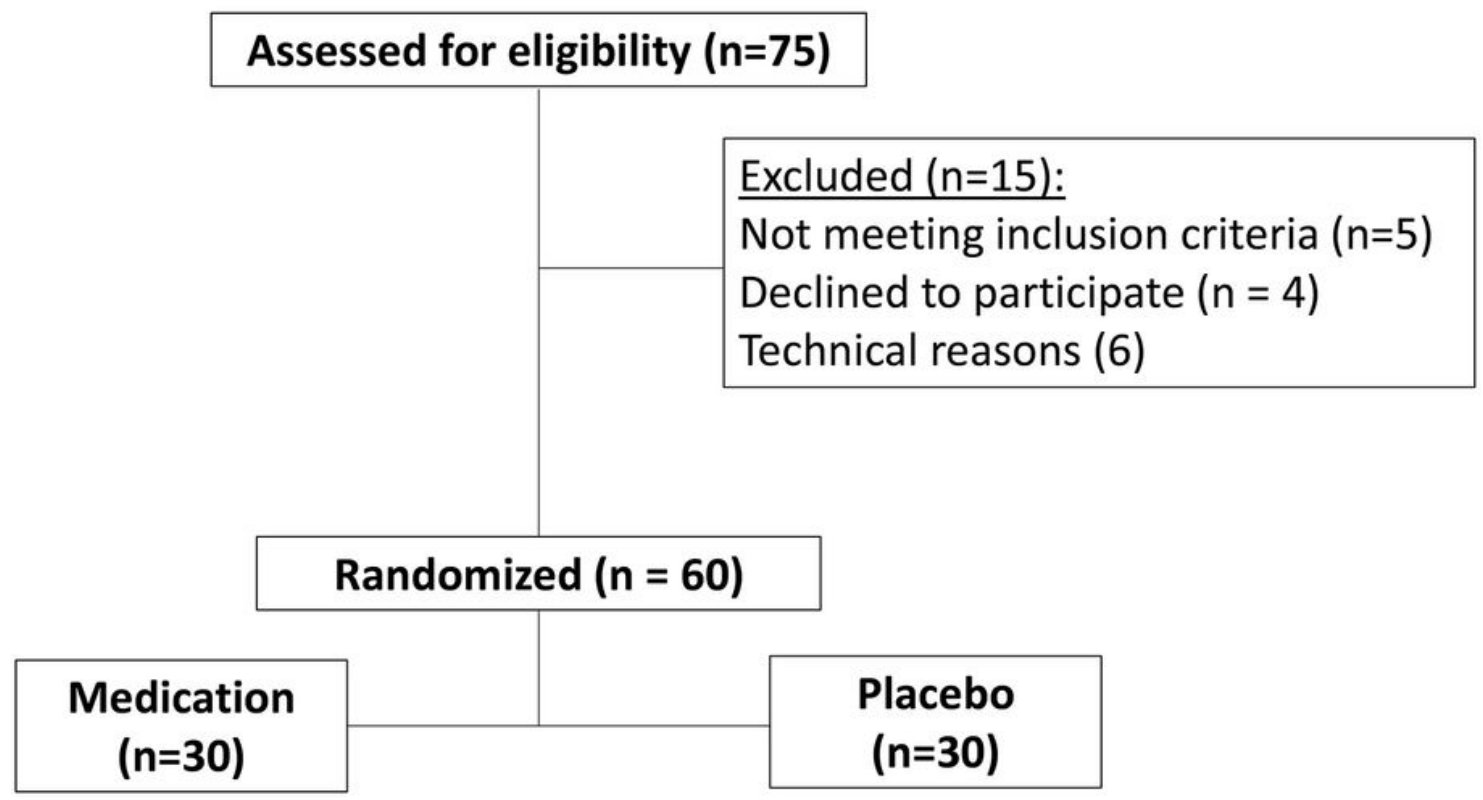

Figure 1

A flow diagram of patients' enrolment 